\title{
Alter
}

Revue de phénoménologie

$21 \mid 2013$

La Vie

\section{La vie du « monde de la vie » : relativité et mobilité}

Julien Farges

\section{OpenEdition}

\section{Journals}

Édition électronique

URL : http://journals.openedition.org/alter/945

DOI : 10.4000/alter.945

ISSN : 2558-7927

Éditeur :

Association ALTER, Archives Husserl (CNRS-UMR 8547)

\section{Édition imprimée}

Date de publication : 1 novembre 2013

Pagination : 73-88

ISBN : 978-2-95-223749-9

ISSN : 1249-8947

\section{Référence électronique}

Julien Farges, «La vie du « monde de la vie » : relativité et mobilité », Alter [En ligne], 21 | 2013, mis en ligne le 01 juin 2019, consulté le 07 juillet 2019. URL : http://journals.openedition.org/alter/945 ; DOI : 10.4000/alter.945

Revue Alter 


\title{
LA VIE DU «MONDE DE LA VIE » : RELATIVITÉ ET MOBILITÉ
}

\author{
Julien Farges
}

On peut dire sans exagération que la vie est chez Husserl le concept à la fois le plus surdéterminé et le plus indéterminé. Il est bien connu, en effet, que la terminologie husserlienne fait place, au cours de son développement, à une prolifération des concepts impliquant une référence à la vie alors même que, pour sa part, «le concept de vie ne fait jamais l'objet d'une thématisation explicite ${ }^{1}$. $C^{\prime}$ est pourquoi, on pourrait être tenté de recourir à la distinction, proposée par Eugen Fink et devenue classique, entre concepts thématiques et concepts opératoires et considérer la vie comme l'un de ces derniers; mais ce serait oublier ce qu'a bien mis en lumière A. Montavont, à savoir que « la vie fait exploser cette distinction opératoire/thématique ». En effet : « La vie est le thème principal du phénoménologue qui cherche à l'expliciter, c'est-à-dire à déterminer de plus en plus précisément ses horizons cachés. Mais, en tant que synthèse jamais achevée, elle est aussi l'opératoire principal du phénoménologue qui part d'elle, de son sol toujours déjà donné $»^{2}$. Ainsi la vie serait-elle cette notion qui ne pourrait être thématisée que comme se dérobant à toute thématisation directe.

Prenant acte de cette situation herméneutique délicate, nous souhaiterions ici interroger ce concept husserlien de vie indirectement, à partir d'un concept qui fonctionne comme son contrepoint, le concept de monde de la vie (Lebenswelt). En posant simplement la question de savoir en quoi consiste cette vie du monde de la vie, ou encore la question de savoir ce que cela signifie pour le monde d'être « de la

\footnotetext{
${ }^{1}$ A. Montavont, De la passivité dans la phénoménologie de Husserl, Paris, PUF, 1999, p. 16.

2 Ibid., resp. p. 45 et 49.
} 
vie », on peut alors espérer approcher certaines déterminations fondamentales du concept husserlien de vie. Nous chercherons ainsi à établir deux point : d'une part, que si les textes husserliens donnent parfois à penser qu'il y a quelque chose comme une vitalité propre au monde de la vie, qu'on peut caractériser par le concept de mouvement, cette mobilité du monde a toujours le sens d'une relativité du monde à la vie du sujet, dont il sert alors de révélateur; et d'autre part, que cette vie du sujet à laquelle le monde de la vie est relatif n'est pas d'abord la vie naturelle du sujet mondain, mais qu'elle n'est pas non plus la seule vie constituante du sujet pur. Ce dont le monde de la vie est corrélatif est plutôt à concevoir comme le mouvement par lequel cette dernière (la vie transcendantale) ne cesse d'entrer en recouvrement avec la première (la vie mondaine), la vie apparaissant dès lors comme la notion où se rejoignent et s'articulent chez Husserl le naturel et le transcendantal. Notre étude partira de l'idée husserlienne assez connue d'une « ontologie du monde de la vie » pour progresser ensuite, sur un mode plus exploratoire, vers des aspects peut-être moins connus de la pensée husserlienne, en tentant $\mathrm{d}^{\prime}$ approfondir à chaque fois le sens qu'il faut donner à la relativité du monde à la vie.

\section{I.}

Comme on le sait, la première façon dont Husserl prétend soumettre le monde de la vie à une thématisation scientifique consiste à en édifier l'ontologie, c'est-à-dire à identifier les structures essentielles et invariantes d'un tel monde. Présentée ainsi, cette tâche peut sembler une simple reformulation du programme de ce que Husserl nomme, dans le sillage des analyses de la $4^{e}$ Recherche logique, l'ontologie matérielle, à savoir le dégagement des structures nécessaires non pas du «quelque chose en général » mais du quelque chose réal, et, par extension, les structures nécessaires de la réalité en général. Pourtant, l'objet de ce premier moment sera de montrer que malgré les apparences, on ne peut pas voir dans l'idée d'une ontologie du monde de la vie le dernier avatar de l'ontologie matérielle et donc que Husserl s'acquitte avec ce projet d'une toute autre tâche.

Pour ce faire, on peut se tourner vers l'une des seules déterminations positives que Husserl fournit dans la Krisis de ce qu'il nomme $l^{\prime}$ «a priori du monde de la vie », à savoir son caractère «subjectif- 
relatif $»^{3}$. Il n'est certes pas aisé de comprendre ce que signifie précisément cette locution husserlienne, mais nous y sommes aidés par Husserl lui-même dans un texte datant vraisemblablement de 1926 où, chose rare, une définition en bonne et due forme est proposée :

On comprend à partir de là le concept authentique du subjectif-relatif. Il inclut tous les prédicats des objets qui, en tant que prédicats d'objets, ne peuvent venir à l'expérience que si des sujets eux-mêmes sont faits ob-jets d'expérience et que ces prédicats sont alors rapportés thématiquement à ces sujets ${ }^{4}$.

Ce qu'on comprend, c'est que s'il y a un a priori du monde de la vie, il ne renvoie pas, en tant $\mathrm{qu}^{\prime}$ a priori subjectif-relatif, à telle ou telle propriété structurale du monde réal ou des choses réales du monde " en elles-mêmes » mais à elles pour autant qu'un sujet s'y rapporte, c'est-à-dire y soit intentionnellement corrélé. Est-ce à dire que la structure du monde de la vie varie d'un sujet (ou d'une communauté subjective) à l'autre? Non, bien entendu, puisque, comme il est bien connu, la mise au jour de cette typique structurale du monde de la vie est destinée par Husserl à la conciliation du relativisme et de l'apriorisme grâce à la reconnaissance phénoménologique du droit du relativisme à l'intérieur de certaines limites irrelatives qui forment quant à elles les contours de cet a priori sui generis ${ }^{5}$. Reste que si la structure générale en laquelle consiste l'a priori du monde de la vie est irrelative, comment comprendre alors que cet a priori soit dit justement subjectif-relatif?

La solution nous semble de considérer qu'un tel a priori ne renvoie pas aux structures invariantes $d u$ monde réal à l'intérieur desquelles son appréhension peut varier mais plutôt aux structures irrelatives de l'avoir-subjectif du monde, qui ne sont rien d'autre, précisément, que les modalités subjectives selon lesquelles l'unique monde pour tous se relativise dans la vie du sujet. Il est clair, dès lors, que l'ontologie du monde de la vie n'est pas superposable à l'ontologie matérielle en tant que doctrine apriorique de la réalité : contrairement à ce que son nom indique au premier abord, cette ontologie du monde de la vie est une façon pour Husserl de se donner à partir du monde une prise

\footnotetext{
${ }^{3}$ E. Husserl, Die Krisis der europäischen Wissenschaften und die transzendentale Phänomenologie. Eine Einleitung in die phänomenologische Philosophie, hrsg. von W. Biemel; 1954 (désormais: Krisis), §36, Hua VI, 143 ; trad. G. Granel, La crise des sciences européennes et la phénoménologie transcendantale, Paris, Gallimard, 1976, p. 159.

${ }^{4}$ E. Husserl, Phänomenologische Psychologie. Vorlesungen Sommersemester 1925, hrsg. von W. Biemel, 1962 (désormais : PP), Beilage VI, Hua IX, 385.

${ }_{5}^{5}$ Cf. E. Husserl, Krisis, § 36, Hua VI, 142 ; trad. cit., p. 158.
} 
descriptive sur la subjectivité et sa vie, dans laquelle « le» monde ne peut pas ne pas se constituer comme «son» monde. L'ontologie du monde de la vie est donc plutôt un moment de ce que Husserl appelle l'ontologie universelle, celle de la subjectivité constituante, et qui était pour lui "la véritable ontologie fondamentale», selon une formule dont la charge polémique semble évidente ${ }^{6}$.

Pour conforter cette lecture, on peut rappeler une thèse que Husserl n'a cessé de formuler à partir de la fin des années $10^{7}$ : à savoir que dans la façon même dont le monde se donne dans l'expérience - c'est-à-dire avec la possibilité essentielle de variations, mais aussi d'erreurs et de corrections - s'annonce de façon nécessaire quoique sur le mode de la présomption un monde "objectif » ou «vrai en soi ». Non pas le monde idéalisé des sciences modernes de la nature, mais plutôt de ce sur quoi fait précisément fond une conception objectivo-théorique $\mathrm{du}$ monde, et qui ne correspond à rien de moins qu'au concept de monde de la vie compris comme unique monde pour tous, dont l'objectivité est le corrélat de l'intersubjectivité universelle exprimée par le pronom «tous». L'idée de Husserl est qu'indépendamment même du type de réalité ou de la teneur réale de ce qui est donné dans l'expérience - question qui intéresse l'ontologie matérielle -, cette expérience, en tant qu'expérience du monde, est indissociablement annonce présomptive $d^{\prime}$ 'un unique monde pour tous et lieu de la relativisation subjective de ce dernier question qui intéresse l'ontologie du monde de la vie. C'est donc bien la vie du sujet qui est le thème de cette ontologie, par suite de quoi les structures qui forment l'a priori du monde de la vie ne peuvent désigner les structures esthétiques de la réalité mondaine mais, en tant que structures subjectives-relatives, les modalités selon lesquelles se relativise subjectivement et nécessairement l'unicité présomptive du monde vrai en soi (du monde pour tous), ou encore ce qu'on pourrait nommer les formes de l'aspectualisation subjective ou de la perspectivation de ce monde unique. La causalité, pour reprendre un exemple husserlien, ne désigne plus dans ce contexte une structure intuitive constitutive de la matérialité chosique mais l'une des modalités selon lesquelles l'unique monde pour tous se diffracte dans des appréhensions subjectives variables : pour l'homme occidental élevé

\footnotetext{
${ }^{6}$ E. Husserl, Die Krisis der europäischen Wissenschaften und die transzendentale Phänomenologie. Ergänzungsband. Texte aus dem Nachlass, 1934-1937, hrsg. von R. N. Smid, 1993 (désormais : Krisis II), Hua XXIX, 151 ; trad. V. Gérard et M. Mavridis in Alter. Revue de phénoménologie, 6, 1998, p. 310 .

${ }^{7}$ Cf. par ex. E. Husserl, $P P, \S 19$, Hua IX, 125 sq. ; trad. Ph. Cabestan, N. Depraz et A. Mazzù, Psychologie phénoménologique (1925-1928), Paris, Vrin, 2001, p. 119 sq.
} 
dans le milieu des sciences modernes de la nature, tel phénomène naturel remarquable sera appréhendé comme l'effet explicable de causes naturelles selon des lois physiques mathématiquement formulables, tandis que le même phénomène sera renvoyé par l'animiste à l'efficacité d'une âme ou d'un esprit animal à même la nature.

Si c'est la vie subjective qui fournit à l'ontologie du monde de la vie son thème véritable, on comprend plus facilement pourquoi Husserl a pu considérer que cette Lebenswelt pouvait fournir le point de départ d'une voie non cartésienne d'accès à la réduction phénoménologique et à l'attitude transcendantale. De même, on comprend aisément pourquoi Husserl a fini par considérer que l'expression même $\mathrm{d}^{\text {" } ~}$ ontologie du monde de la vie » est insatisfaisante, et finalement absurde (widersinnig), point souvent méconnu par les commentateurs alors que Husserl est pourtant catégorique :

\begin{abstract}
À la vérité, l'expression "ontologie du monde de la vie »-[pour] nous qui avons constamment compris sous l'öv l'objectivement étant, précisément, et [pour qui] tout a priori avait donc lui-même un sens objectif - est une expression absurde. Il s'agit justement d'une de ces métamorphoses radicales des concepts que comporte le passage à la dimension transcendantale.
\end{abstract}

Mais surtout, si l'ontologie du monde de la vie consiste à ressaisir la relativité du monde au sein d'une forme qui serait celle de la relativisation elle-même (à l'opposé de l'ontologie physico-mathématique des sciences modernes qui peut être décrite comme une façon d'irrelativiser le monde en court-circuitant sa relativité subjective), elle revient à inscrire la relativité des appréhensions du monde dans le sens constitutif du monde de la vie lui-même, sous le titre d'une relativité transcendantale qu'il faut bien distinguer de la simple relativité empirique $\mathrm{du}$ monde induite par la variété factuelle des horizons culturels au sein desquels il est aperçu. La relativité qui est ici en question porte sur la validité du monde, il s'agit d'une Geltungsrelativität renvoyant comme telle à deux caractéristiques eidétiques fondamentales et inséparables : d'une part, au fait que l'unité de ce monde est corrélative non seulement à la multiplicité ouverte des sujets auxquels il apparaît, mais aussi, pour chaque sujet, à une infinité de modes possibles de donation dans la synthèse ouverte desquels il se constitue ${ }^{9}$; d'autre part, au fait que l'expérience est une

\footnotetext{
${ }^{8}$ E. Husserl, Krisis II, Hua XXIX, 173, n. 1.

${ }^{9}$ Cf. par ex. E. Husserl, Die Lebenswelt. Auslegungen der vorgegebenen Welt und ihrer Konstitution. Texte aus dem Nachlass (1916-1937), hrsg. von R. Sowa, 2008 (désormais : Lebenswelt), Hua XXXIX, 690: «Il est clair que ce monde conscient est le monde qui, à chaque fois, nous apparaît
} 
structure de concordance qui se maintient en se rétablissant dans et à travers un procès de correction permanente et qu'elle ne peut donc, comme telle, être le lieu de constitution d'aucune validité absolue et définitive mais toujours seulement relative et présomptive. La relativité du monde de la vie désigne ainsi l'instabilité constitutive de la synthèse intentionnelle dont il est le corrélat et en dehors de laquelle il n'aurait aucune validité ni aucun sens, et c'est elle qui appelle régulièrement sous la plume de Husserl une référence à Héraclite pour décrire la forme de la conscience intentionnelle dont le monde de la vie est le corrélat ${ }^{10}$, le monde de la vie pouvant dès lors lui-même être caractérisé comme "monde du flux héraclitéen ${ }^{11}$. Or cette référence n'est pas anodine: en renvoyant, comme le faisait Platon, le relativisme à la doctrine du philosophe $\mathrm{d}^{\prime}$ Éphèse ${ }^{12}$, Husserl laisse en effet entendre que le concept de mouvement serait adéquat pour rendre compte de la relativité transcendantale du monde de la vie. Et c'est un fait qu'il affirme lui-même qu'en raison de la structure de concordance selon laquelle le monde est prédonné dans l'expérience, "le monde de la vie est toujours dans un mouvement qui est celui d'une constante relativité dans la validité (Geltungsrelativität) »13.

Si l'on cherche à présent à décrire phénoménologiquement par un seul concept cette relativité du monde de la vie, c'est-à-dire la modalité la plus spécifique de sa validité transcendantale, il semble que le concept le plus approprié soit la Jeweiligkeit, qui, comme souvent chez Husserl, est partout opératoire à partir du milieu des années 20 bien qu'il ne fasse jamais, à notre connaissance l'objet, d'une thématisation explicite. Ramené à sa signification fondamentale, ce concept exprime moins une périodicité intra-mondaine ${ }^{14}$ que quelque chose comme la distributivité de la validité du monde dans la vie intentionnelle. Si l'on s'attache en effet tout d'abord à cette relativité transcendantale selon laquelle le monde ne vaut que dans le mouvement d'incessante correction de l'expérience qu'en fait le sujet, alors «il est clair que ce monde conscient est le monde qui est à chaque fois apparaissant subjectivement ainsi et ainsi, à chaque fois valant ainsi et

\footnotetext{
subjectivement de telle ou telle manière, valant de telle ou telle manière. [...] Si nous en restons à la teneur de validité de la vie présente, alors nous sommes dans un changement et un relativisme incessants. Sous le titre "monde" se tient quelque chose qui est toujours autre » (nous soulignons).

${ }^{10}$ Cf. E. Husserl, Krisis, Hua VI, 158 sq., 173, 181, 357 (trad. cit., p. 177, 193, 202, 394).

${ }^{11}$ E. Husserl, Lebenswelt, Hua XXXIX, 690.

12 Cf. Platon, Théétète, 152e, 160d, 179d-e ; Cratyle, 401d-e, 411b-c, 440a-c.

${ }^{13}$ E. Husserl, Krisis, Beilage XVIII, Hua VI, 465 ; trad. cit. (modifiée), p. 515 (nous soulignons).

${ }^{14} \mathrm{C}^{\prime}$ est là le sens que $\mathrm{K}$. Held donne à ce concept dans son ouvrage Heraklit, Parmenides und der Anfang von Philosophie und Wissenschaft. Eine phänomenologische Besinnung, Berlin/New-York, Walter de Gruyter, 1980, p. 221 sq., 295 sq.
} 
ainsi $»^{15}$. Ce qui se constitue comme monde dans le changement permanent des modes subjectifs est ainsi ce que Husserl nomme "l'être pour nous d'à chaque fois » ${ }^{16}$, si bien que le monde prédonné "est justement monde chaque fois dans ses "modes d'apparition", dans les aperceptions, ouvert à d'autres modifications qui en changeront la forme, qui en changeront éventuellement l'être en non-être (illusion), modifications à travers lesquelles, bien que par corrections, se produit malgré tout l'unité d'un seul monde qui continue à valoir comme étant $[\ldots] »^{17}$. Le monde est donc «toujours un autre » dans la vie et c'est évidemment sur cette relativité que se fonde ensuite la relativité au sens historico-culturel, qui n'en est qu'une manifestation particulièrement prégnante. Le rôle du concept d'Umwelt est dès lors de désigner quel monde relatif la Lebenswelt est à chaque fois.

La Jeweiligkeit apparaît ainsi comme une détermination fondamentale du monde de la vie compris non pas seulement au sens objectif comme monde d'une expérience naturelle préscientifique ni comme omnitudo realitatis, mais au sens subjectif d'un monde prédonné au sein de l'enchaînement des prestations constituantes de la vie transcendantale du sujet. Pour reprendre une expression remarquable de Husserl, le monde de la vie est bien en ce sens le monde dans la vie ${ }^{18}$, et ce dans une "appartenance intentionnelle » si étroite qu'elle vire parfois, sous sa plume, à la pure et simple identification. Ainsi se risque-t-il à écrire en 1933: "Le monde désigne une faculté de faire systématiquement l'expérience et [de] confirmer par les moyens de l'expérience le sens d'être identique ${ }^{19}$. Or si l'étude systématique de cette inscription subjective du monde est la tâche de l'ontologie du monde de la vie, de la Lebenswelt, elle consiste donc très exactement à mettre au jour la vie subjective comme Weltleben, vie de monde, se laissant d'abord décrire comme une puissance de relativisation, selon une détermination qui situe la phénoménologie husserlienne dans une proximité inattendue, peut-être, avec Nietzsche ${ }^{20}$.

\footnotetext{
${ }^{15}$ E. Husserl, Lebenswelt, Hua XXXIX, 690 (nous soulignons).

${ }^{16}$ Ibid., Hua XXXIX, 449 (nous soulignons).

17 E. Husserl, Krisis, Abhandlung II, Hua VI, 299 ; trad. cit., p. 330 (nous soulignons).

${ }^{18}$ Cf. E. Husserl, Lebenswelt, Hua XXXIX, 690: "C'est là le monde dans la vie subjective ellemême, le monde de la vie, comme nous pourrions l'appeler ».

${ }_{19}$ E. Husserl, Zur Phänomenologie der Intersubjektivität. Dritter Teil : 1928-1935, hrsg. von I. Kern, 1973 (désormais : ZPI-III), Hua XV, 621 ; trad. R. Brandmeyer, Ph. Cabestan, N. Depraz et A. Montavont in Alter. Revue de phénoménologie, 3, 1995, p. 213 (nous soulignons).

${ }^{20} \mathrm{Cf}$. F. Nietzsche, Par-delà bien et mal. Prélude à une philosophie de l'avenir, trad. C. Heim sur le texte de l'édition de G. Colli et M. Montinari, CEuvres philosophiques complètes, VII, Paris, Gallimard, 1971, Préface, p. 18 : «le perspectivisme, condition fondamentale de toute vie».
} 
Toutes ces analyses montrent donc que le concept de Lebenswelt est le lieu chez Husserl d'une sorte de détermination réciproque de l'être du monde et de la vie du sujet, dans laquelle les concepts de relativité et de mobilité sont essentiels : le monde de la vie reçoit le caractère de la relativité et de la mobilité en tant que monde dans une vie qu'il révèle en retour comme puissance de relativisation ou d'animation. Dans la deuxième moitié de cette étude, nous souhaiterions montrer que ces deux notions connaissent chez Husserl un deuxième niveau, plus radical, de détermination, auquel correspond une compréhension elle-même plus précise du concept husserlien de " monde de la vie ».

II.

Ce second niveau d'analyse apparaît rapidement pour peu qu'on se rappelle que le monde de la vie n'est pas et n'a jamais été pour Husserl un monde naturel au sens d'un monde purement perceptif, mais au contraire toujours le monde socio-historique de la culture. Or ce dernier est un monde imprégné de signification car peuplé d'objets signifiants (des institutions, des œuvres, des traditions, des théories...), dont la constitution renvoie à des activités subjectives productrices de sens ou à des actes instituteurs de signification qui ajoutent aux purs substrats perceptifs et naturels une couche prédicative spécifique (celles des Bedeutungsprädikate). Comme on le voit, cette activité subjective renvoie à un concept de vie totalement débiologisé, ainsi que Husserl le souligne lui-même dans la Conférence de Vienne de mai 1935: "Le mot vivre n'a pas ici le sens physiologique, il signifie une formation téléologique, spirituelle, de la vie prestative: au sens le plus vaste, de la vie qui crée la culture dans l'unité d'une historicité $»^{21}$. Mais le point important pour nous est qu'une fois ces prédicats de significations institués, ils sont constamment aperçus ou co-appréhendés avec les étants qu'ils déterminent culturellement, sans qu'il soit nécessaire de réitérer l'institution et donc en une sorte de nouvelle immédiateté : l'outil est immédiatement saisi dans son utilité et non comme une simple chose ayant par ailleurs telle ou telle fonction utile. Dans sa terminologie, Husserl dira que les prédicats de significations sont institués en tant que "validités-pour » (Geltungenfür) le monde de la vie, au sens où ils sont destinés à être appré-

\footnotetext{
${ }^{21}$ E. Husserl, «La crise de l'humanité européenne et la philosophie », in Krisis, Hua VI, 315 ; trad. cit., p. 348 .
} 
hendés comme l'étoffe même de ce monde. Corrélativement, le monde de la vie sera dit "accueillir en lui » (in sich aufnehmen) ces validités signifiantes qui se sont donc «ajoutées en dépôt» (sich zugeschlagen) à sa teneur propre si bien qu'elles ont à proprement parler " pénétré » en lui (eingegangen sind). Toutes ces tournures verbales peuvent en réalité être considérées comme des formes d'un unique concept husserlien dont le rôle est absolument central pour la compréhension de la phénoménologie du monde de la vie, concept fixé fort tardivement mais dont les racines plongent dans la phénoménologie génétique des années 20 - le concept d'Einströmen, qu'on ne peut guère traduire autrement que par «afflux». Comme le montrent les quelques occurrences de ce concept dans la Krisis ${ }^{22}$, il désigne l'incorporation permanente du prédicatif (qu'il soit théorique, axiologique ou pratique), bref de tout ce qui ressortit à la validité médiate ou à la signification, dans le flux de l'expérience vitalo-mondaine antéprédicative, incorporation qu'on pourrait donc décrire comme un mouvement d'immédiation permanente du médiat ou de devenir intuitif du signitif.

Il convient ici de rappeler que c'est par le moyen de ce concept que Husserl n'en reste pas, dans la Krisis, à la mise en scène d'une opposition stérile ou d'un contraste pur et simple entre monde de la vie et monde scientifique mais parvient au contraire à suspendre ce contraste dans ce qu'il nomme le "paradoxe du rattachement mutuel d'un "monde objectivement vrai" et d'un "monde de la vie" ${ }^{23}$, à savoir le paradoxe d'un monde qui est intuitif précisément en ce qu'il est lesté de sédiments originellement non-intuitifs. Dans la mesure où la science représente une certaine activité culturelle et productrice de signification dans le monde de la vie, elle n'est pas seulement référée à lui comme à un sol originaire de confirmation et de validité, mais elle lui est pour ainsi dire également destinée comme au milieu dans lequel elle est appelée à devenir disponible à son tour comme objet d'une appréhension immédiate, d'une croyance naturelle ou comme point de départ pour des démarches théoriques ultérieures ${ }^{24}$. C'est ainsi que notre monde de la vie, à nous Européens modernes ou postmodernes, est pour ainsi dire un monde galiléen, non pas au sens où il serait le monde de l'attitude théorique des physiciens mais au sens où les théories de la physique moderne se sont incorporées à notre

${ }_{22}$ Cf. Ibid., Hua VI, 115, 141 n., 213, 462, 466 ; trad. cit., p. 129, 157 n., 237, 511, 518.

${ }^{23} \mathrm{Ibid}$., § 34 e, Hua VI, 134 ; trad. cit. p. 149.

${ }^{24}$ Cf. ibid. 
façon de nous rapporter immédiatement au monde et à ses objets dans l'attitude naturelle.

Cela rappelé, il faut souligner que cette notion d'afflux, par laquelle Husserl rend compte de l'incorporation permanente du prédicatif à l'antéprédicatif, est intimement reliée à celle de mouvement et conduit Husserl, à travers l'idée d'une Weltbewegung, à attribuer la mobilité au monde lui-même, comme si la subjectivité intentionnelle, par ses actes constitutifs de signification, communiquait sa vie au monde qu'elle constitue comme son monde de la vie. Ainsi Husserl écrit-il :

le monde de la vie est pour nous le monde [...] dans un mouvement permanent $d u$ fait que toute nouvelle activité produit de nouvelles composantes de sens qui adviennent de manière simplement aperceptive au monde, prédonné jusqu'alors en un seul sens d'être ${ }^{25}$.

Le concept de mobilité désigne ici tout d'abord cette instabilité du monde, cette augmentation permanente de sa teneur phénoménologique qui a pour origine l'incessant et inévitable afflux en lui de ce qui a été élaboré dans toutes les formes de pratique subjectives institutrices de sens. Il est en ce sens un monde "toujours nouveau ", c'est-à-dire que rien ne peut être perçu de lui sans que soit aperçu en même temps un halo toujours plus large de significations concrètes. Mais la Weltbewegung ne désigne pas seulement cet enrichissement en significations que Husserl nomme des acquis (Erwerbe) aperceptifs. En effet, contrairement à ce qu'on pourrait croire au premier abord, ce terme $d^{\prime}$ " acquis » ne doit pas être entendu comme le résultat stable d'un processus d'acquisition lui-même achevé, mais un terme relatif au sein d'un processus d'acquisition déjà inauguré et toujours en cours. On peut donc dire que le monde de la vie est en mouvement permanent dans la mesure où il n'est donné intuitivement qu'au sein $\mathrm{d}^{\prime}$ horizons aperceptifs mouvants et donc au sens où il est pris dans le flux d'une acquisition subjective permanente. En réalité, le monde de la vie n'est pas un donné qui pourrait être acquis une fois pour toutes mais il est plutôt l'horizon d'une acquisition subjective et intersubjective en droit infinie: il est prédonné non seulement lesté de couches aperceptives qui ont déjà été acquises subjectivement mais aussi comme matériau pour de nouvelles acquisitions subjectives destinées à affluer en lui comme autant de nouveaux sédiments aperceptifs ${ }^{26}$. En ce sens, le monde de la vie est un phénomème constitutivement inachevé et

\footnotetext{
${ }^{25}$ E. Husserl, Lebenswelt, Hua XXXIX, 516 ; cf. aussi p. 514.

${ }^{26}$ Cf. ibid., Hua XXXIX, 517.
} 
inachevable, pris dans le mouvement d'une tradition subjective, en l'occurrence celui de la réacquisition vivante des significations déjà instituées, de leur transmission intersubjective et de l'implication de nouvelles acquisitions possibles de signification.

Comme on le voit, à travers cette mobilité du monde c'est aussi l'idée d'une «histoire du monde ${ }^{27}$ qui prend corps, c'est-à-dire la thèse selon laquelle le monde de la vie est intrinsèquement historique. Reste que cette historicité ne saurait alors renvoyer à la succession des événements factuels, mais plutôt à cette variabilité et à cette mobilité en vertu desquelles il est impossible de décrire le monde de la vie dans sa teneur concrète sans le décrire simultanément comme étant pris dans une incessante tradition subjective de sens. Il convient donc de souligner ici que c'est à l'Einströmen, au mouvement de l'afflux, que le monde de la vie doit de recevoir non seulement une épaisseur concrète mais aussi un dynamisme ou une vitalité historiques.

Or il faut ici ajouter que le concept d'Einströmen est aussi mobilisé par Husserl dans les années 30 pour décrire ce que Fink avait pour sa part nommé dans la Sixième Méditation cartésienne la "mondanéisation » du transcendantal: à savoir le processus, assez délicat à décrire, au terme duquel, d'une part, la subjectivité transcendantale constituante s'aperçoit et se "sait » elle-même comme subjectivité psychologique personnelle localisée dans le monde, et au terme duquel d'autre part les acquis théoriques de la phénoménologie transcendantale (les descriptions concrètes, les concepts descriptifs, la réduction elle-même) apparaissent dans le monde comme une science susceptible d'être apprise, mise en œuvre, discutée ou prolongée. L'enjeu de cette thématique de la mondanéisation est considérable puisqu'il s'agit de résorber la coupure apparente entre transcendantalité et naturalité en montrant que rien de ce qui est transcendantal n'est en définitive étranger au monde naturel puisqu'il finit nécessairement par y affluer, c'est-à-dire s'y localiser et s'y rendre aperceptible, qu'il s'agisse de l'ego transcendantal lui-même ou de tel ou tel élément doctrinal de la phénoménologie en tant que science transcendantale.

Or, que ce phénomène de l'afflux du transcendantal dans le monde soit de première importance eu égard à notre étude de la relativité et de la mobilité du monde de la vie, c'est ce que montrent ces lignes de Husserl :

\footnotetext{
${ }^{27}$ E. Husserl, Krisis, Beilage XXV, Hua VI, 501 ; trad. cit., p. 555. Cf. également L'Origine de la géométrie, in ibid., Beilage III, Hua VI, 378 ; trad. cit., p. 418.
} 
C'est donc dans un devenir vivant, dans un changement incessant des modes subjectifs que le monde étant se constitue pour nous en tant que sens d'être [...]. Mais comment peut-il, comment le monde étant pour nous peut-il avoir "en " nous une genèse, un devenir subjectif? N'est-ce pas là une supposition contradictoire? Ne sommes-nous pas nous-mêmes dans le monde, nous les réalités humaines, charnellement et psychiquement, avec toute notre vie de conscience, toutes nos propriétés subjectives, nos habitualités, nos visées et ce que nous y pouvons viser ?28

Si nous le comprenons bien, ce texte de Husserl veut dire que la mise en évidence de la relativité transcendantale du monde de la vie, sur laquelle débouchait son ontologie, n'est qu'un premier pas dans la tâche d'une genèse transcendantale du monde. En effet, si l'ontologie du monde de la vie pouvait commencer à rendre compte de l'inscription du monde dans la vie du sujet à titre de phénomène de validité, elle fait en revanche l'impasse complète sur l'inscription symétrique et inverse, celle de la subjectivité transcendantale dans le monde qu'elle constitue.

Autrement dit, il faut ajouter à la relativité du monde de la vie que nous avons identifiée précédemment une deuxième forme de relativité : le monde de la vie n'est pas seulement relatif aux opérations constituantes de la subjectivité transcendantale mais il forme aussi l'horizon de la subjectivité mondaine, c'est-à-dire humaine ou personnelle. Or puisque nous avons rappelé à l'instant que cet ego humain est lui-même un acquis transcendantal, qu'il est lui-même pris dans un mouvement d'acquisition ou encore que sa constitution a la forme d'une genèse, il faut dire que le monde de la vie est également relatif au mouvement par lequel la subjectivité transcendantale ne cesse de s'inscrire dans le monde qu'elle constitue, c'est-àdire au mouvement par lequel elle ne cesse, par auto-aperception, de faire sa propre acquisition en tant que subjectivité humaine et mondaine.

Comment clarifier ces formules qui semblent dangereusement spéculatives et comment rendre ainsi compréhensible cette double relativité $\mathrm{du}$ monde de la vie? On peut tout d'abord s'appuyer sur le concept d'acquis. En effet, si le monde de la vie n'est pas un acquis parmi d'autres de la subjectivité constituante mais, comme le dit Husserl, son « acquis universel » $c^{\prime} e s t$, comme il le précise également, parce qu'il ne désigne rien d'autre finalement que la forme ontologique (isolable par l'ontologie du monde de la vie, précisément) du

28 E. Husserl, Lebenswelt, Hua XXXIX, 448-449. 
tout de l'acquis ${ }^{29}$. Or ce "tout de l'acquis » inclut l'ego humain luimême dans la mesure où il est le résultat du processus de mondanéisation qui accompagne nécessairement toute constitution. Cela revient donc très exactement à dire que le sujet ne peut s'apercevoir luimême et ne se constituer dans toute son épaisseur concrète que sur fond de monde. Par conséquent, on peut expliciter cette double relativité transcendantale du monde de la vie en y voyant la façon dont Husserl cherche à rendre compte de ce qu'il conviendrait de nommer l'entre-appartenance $d u$ monde et $d u$ sujet, ou encore, dans le contexte d'une phénoménologie génétique, leur co-originarité : la subjectivité « dans » la vie de laquelle se constitue le monde (première relativité) s'aperçoit elle-même localisée "dans » le monde qu'elle constitue et qui forme son horizon (seconde relativité). Ce serait donc à la thématisation rigoureuse et à la description précise de cette entre-appartenance que tendrait la phénoménologie du monde de la vie en son sens le plus large.

On peut trouver une confirmation de cette lecture dans une certaine porosité terminologique en vertu de laquelle il arrive, sous la plume de Husserl, que chacun des deux termes reçoive des déterminations descriptives de l'autre. Si c'est bien connu dans le cas de la subjectivité qui, mondanéisée, est descriptible comme un "morceau du monde $»^{30}$, c'est moins connu dans le cas du monde de la vie luimême, auquel Husserl n'hésite pourtant pas à attribuer plus d'une fois le caractère du flux (Strömen), c'est-à-dire rien de moins que la forme même de la vie subjective. Et de fait Husserl ne se contente pas d'évoquer " le monde existant pour nous dans le flux changeant de ses modes de donnée ${ }^{31}$ (comme au premier sens de la relativité du monde de la vie), mais il lui arrive de parler aussi du «flux entier du monde de la vie ${ }^{32}$. Cette tendance culminera au moment où, pour désigner le monde compris comme horizon de l'inscription mondaine de la subjectivité constituante, Husserl ira jusqu'à substituer à l'expression "monde de la vie » (Lebenswelt) celle de "vitalo-mondanéité fluante»(strömende Lebensweltlichkeit) ${ }^{33}$. Si l'on peut, comme C. Bermes, voir dans cette désignation la formulation la plus aboutie

\footnotetext{
${ }^{29}$ Ibid., Beilage XX, Hua VI, 471 ; trad. cit., p. 522-523.

${ }^{30}$ Ibid., § 29, Hua VI, 115-116; trad. cit., p. 129.

31 Ibid., § 29, Hua VI, 115 ; trad. cit., p. 129.

32 Ibid., § 63, Hua VI, 223 ; trad. cit., p. 248.

${ }_{33}$ Cf. E. Husserl, Krisis II, Hua XXIX, 313, où il est question plus précisément du «flux de la vie humaine universelle et de sa vitalo-mondanéité fluante» («Strom des universalen Menschheitsleben und seiner strömenden Lebensweltlichkeit »).
} 
du concept de monde de la vie ${ }^{34}$, c'est parce qu'elle soustrait complètement le mode d'être du monde à celui des objets du monde tout en laissant entendre que ce monde est bien subjectivement constitué. Cette expression permet également de comprendre que le monde de la vie n'est certes pas simplement le monde de l'attitude naturelle mais qu'il n'est pas non plus un simple phénomène de validité dans la vie transcendantale d'une subjectivité démondanisée ; il est plutôt le corrélat du mouvement par lequel cette subjectivité ne cesse d'entrer en recouvrement avec la subjectivité humaine et mondaine, c'est-à-dire le corrélat de l'unité des deux sens de la vie, naturelle et transcendantale, unité dynamique et toujours en cours $\mathrm{d}$ 'acquisition. Ainsi s'expliquerait également la déclaration frappante de Husserl dans la Krisis selon laquelle «le traitement théorique radical du monde est le traitement interne systématique et pur de la subjectivité s' "extériorisant" elle-même dans l'extérieur »35.

Une dernière façon d'éclairer la relativité du monde à cette extériorisation de la subjectivité transcendantale dans le monde consisterait à valoriser le fait que ce mouvement d'auto-acquisition aperceptive du sujet est celui d'une humanisation et à interpréter par conséquent la mobilité et la vitalité historiques du monde de la vie précisément comme une dynamique d'humanisation. La constitution transcendantale apparaîtrait ainsi comme le processus d'humanisation corrélative du sujet et du monde qui est à chaque fois le sien, ce qui reviendrait en fait à introduire une dimension anthropologique dans ce que Husserl nomme par ailleurs "l'a priori de l'histoire». Les textes husserliens tardifs montrent en effet que le devenir historique du monde de la vie dans la traditionnalité des institutions transcendantales ne saurait être pensé indépendamment du devenir historique effectif des hommes dans le monde qui est à chaque fois le leur : il appartient a priori au monde de la vie, tel qu'il est transcendantalement constitué, d'être un monde dans lequel des hommes y vivent une histoire humaine. De sorte qu'il faut reconnaître à la fois que l'humain est essentiellement un être historique ${ }^{36}$ - ou encore que son concept est un concept historique ${ }^{37}$ - et que toute histoire est non

\footnotetext{
${ }^{34}$ Cf. C. Bermes, "Welt" als Thema der Philosophie. Vom metaphysischen zum natürlichen Weltbegriff, Hamburg, Felix Meiner Verlag, 2004, p. 227, n. 80.

${ }^{35}$ E. Husserl, Krisis, § 29, Hua VI, 116 ; trad. cit., p. 129.

${ }^{36}$ Cf. E. Husserl, ZPI-III, Hua XV, 391 ; trad. N. Depraz, Sur l'intersubjectivité II, Paris, PUF, 2001, p. 323.

${ }^{37}$ Cf. E. Husserl, Krisis II, Hua XXIX, 172: "Le "concept" d'homme n'est pas un concept ontologique mais avant tout un concept historique» ("Der "Begriff" des Menschen ist kein ontologischer, sondern zunächst ein historischer Begriff »).
} 
seulement une histoire essentiellement humaine mais surtout mouvement d'humanisation corrélative de l'humanité et du monde qui est le sien. Quant aux modalités selon lesquelles est accomplie cette formation humanisante du monde, elles sont rassemblées sous le concept englobant de culture, qui désigne à la fois l'effort et le résultat de l'effort permanent d'inscription dans le monde des fins humaines ${ }^{38}$, effort par lequel le monde ne cesse de prendre un visage toujours plus humain, selon une image à laquelle Husserl recourt volontiers ${ }^{39}$.

Il n'y a donc pas de reconnaissance de l'historicité humaine dans le monde qui ne soit simultanément reconnaissance de l'historicité humaine $d u$ monde - telle parait être la conclusion à laquelle parvient ici Husserl. Elle signifie en particulier que l'a priori du monde de la vie ne saurait être défini seulement comme un a priori historique, mais plutôt comme un a priori historico-anthropologique ${ }^{40}$, et que l'ontologie du monde de la vie doit en définitive coïncider avec l'anthropologie. Cette dernière conclusion est explicitement tirée par Husserl dans un manuscrit remarquable de septembre 1932, qui nous permettra de renouer en fin de parcours avec le thème de nos premières remarques :

Il s'agirait donc d'une ontologie du monde en tant que monde d'expérience possible ou, ce qui est équivalent, d'un monde qui est un monde humain et qui, pour les hommes et les humanités entrant en communication, doit pouvoir être constitué et connaissable comme le même in infinitum, malgré toutes les corrections et les élargissements complémentaires individuels et réciproques. Cette ontologie serait donc une anthropologie apriorique ${ }^{41}$.

$\mathrm{Si}$, en guise de conclusion, nous rassemblons brièvement les acquis de ce parcours dans la phénoménologie husserlienne du monde de la vie, le premier point à souligner est que la Lebenswelt y fonctionne comme un révélateur de la vie de la conscience intentionnelle, de sorte que la science du monde de la vie, qu'elle soit élaborée dans une perspective ontologique ou dans une perspective transcendantale, est toujours en définitive un moment de l'auto-thématisation réflexive de

\footnotetext{
${ }^{38}$ Cf. F. Dastur, Husserl. Des mathématiques à l'histoire, Paris, PUF, 1995, p. 109 sq.

${ }^{39}$ Cf. E. Husserl, Lebenswelt, Hua XXXIX, 510.

${ }^{40}$ Cf. E. Husserl, Krisis II, Hua XXIX, 316 : « Les hommes appartiennent à la structure du monde. Eux aussi ont part à l'a priori universel. [...] En tant que science, l'histoire peut recevoir la nouvelle forme d'une direction par l'a priori. Il en résulte une anthropologie scientifique, et certes de l'homme personnel dans toutes ses possibilités et ses spécificités invariantes, mais aussi, à partir de là, de l'homme et de l'humanités factices » (nous soulignons).

${ }^{41}$ E. Husserl, Lebenswelt, Hua XXXIX, 57 (nous soulignons).
} 
la subjectivité. C'est pourquoi nous avons vu que Husserl n'hésitait pas à attribuer à ce monde de la vie des déterminations qui reviennent par principe à la subjectivité intentionnelle (faculté de viser l'identique ou forme de flux) ou encore à lui attribuer une mobilité vitale qui est en définitive celle du sujet intentionnel. Dans l'expression de "monde de la vie ", le génitif doit donc être entendu en son sens subjectif. Mais on aurait tort de croire pour cette raison que le monde de la vie serait le concept par lequel s'accomplit chez Husserl la dissolution idéaliste $\mathrm{du}$ monde effectif dans les replis d'une subjectivité aussi démiurgique que démondanisée. Si la vie du monde de la vie, la vie révélée par lui, est bien "vie de monde » au sens de l'enchaînement des actes intentionnels d'un sujet transcendantal qui constitue le monde en le relativisant à chaque fois comme son monde, elle est également et simultanément vie dans le monde qui est ainsi constitué. Ainsi, le concept de monde de la vie est destiné prioritairement par Husserl à rendre compte descriptivement de l'entreappartenance du sujet et du monde, si bien que la vie elle-même, en tant qu'intentionnelle, demeure toujours chez Husserl vie $d u$ sujet. On peut bien sûr le déplorer en y voyant quelque chose comme une captation de la vie par la subjectivité, révélant l'incapacité husserlienne à prendre la mesure des ressources philosophiques du concept de vie. Mais il nous semble qu'on peut aussi considérer que c'est définir la vie de façon consistante que d'y voir la puissance de façonnement et d'acquisition réciproque d'un sujet et de son monde. Outre qu'elle permet, dans le sillage de la biologie des milieux, une approche renouvelée de l'animal et de son monde sous une catégorie élargie de la subjectivitét2, cette détermination de la vie donne surtout une justification et un contenu au projet husserlien volontairement paradoxal d'une «philosophie scientifique de la vie ${ }^{43}$ : faire droit à la vie, c'est-à-dire à la relativité et à la mobilité, pour décrire les rapports du sujet humain au monde sans céder pour autant aux tendances sinon irrationalistes du moins anti-théoriques de la Lebensphilosophie mais en s'efforçant au contraire de concilier ces notions avec une perspective aprioriste et fondationnaliste susceptible de renouveler le programme d'une anthropologie philosophique.

\footnotetext{
42 Pour une exploitation récente de la phénoménologie husserlienne dans cette direction, cf. F. Burgat, Une autre existence. La condition animale, Paris, Albin Michel, 2012, chap. 7, p. 243 sq.

${ }^{43}$ E. Husserl, Natur und Geist. Vorlesungen Sommersemester 1927, hrsg. von M. Weiler, 2001, Hua XXXII, 241.
} 